\title{
SINGULAR MOSER-TRUDINGER INEQUALITY WITH THE EXACT GROWTH CONDITION IN $\mathbb{R}^{n}$
}

\author{
ZHAO LIU AND LU CHEN
}

Abstract. In this paper, we establish the singular Moser-Trudinger inequality with exact growth condition. We prove that there exists a positive constant $C_{n}$ such that

$$
\int_{\mathbb{R}^{n}} \frac{\Phi\left(\alpha_{n}\left(1-\frac{t}{n}\right)|u|^{\frac{n}{n-1}}\right)}{|x|^{t}(1+|u|)^{\frac{n}{n-1}}} d x \leqslant C_{n} \int_{\mathbb{R}^{n}} \frac{|u(x)|^{n}}{|x|^{t}} d x
$$

for all $u \in W^{1, n}\left(\mathbb{R}^{n}\right)$ with $\int_{\mathbb{R}^{n}}|\nabla u|^{n} d x \leqslant 1$, where $\Phi(t):=e^{t}-\sum_{i=0}^{n-2} \frac{t^{i}}{i !}$. In order to avoid using symmetry and rearrangement, we employ the change of variables developed by Dong and Lu in [14] (see also Lam and Lu in [16]) to transform the singular Moser-Trudinger inequality with the exact growth condition to the corresponding non-singular case.

Mathematics subject classification (2010): 35A23, 42B37.

Keywords and phrases: Change of variables, Sharp constants, singular Moser-Trudinger inequality with the exact growth condition, Sobolev space.

\section{REFERENCES}

[1] D. Adams, A sharp inequality of J. Moser for higher order derivatives, Ann. of Math. 128, 2 (1988), 385-398.

[2] Adimurthi AND K. SANDEeP, A singular Moser-Trudinger embedding and its applications, NoDEA Nonlinear Differential Equations Appl. 13, 5 (2007), 585-603.

[3] S. AdAchi AND K. TANAKA, Trudinger type inequalities in $\mathbb{R}^{N}$ and their best exponents, Proc. Amer. Math. Soc. 128, 7 (2000), 2051-2057.

[4] Adimurthi AND Y. YAng, An interpolation of Hardy inequality and Trundinger-Moser inequality in $\mathbb{R}^{N}$ and its applications, Int. Math. Res. Not. 13 (2010), 2394-2426.

[5] W. BECKNER, Sharp Sobolev inequalities on the sphere and the Moser-Trudinger inequality, Ann. of Math. 138, 1 (1993), 213-242.

[6] T. Branson, L. Fontana And C. Morpurgo, Moser-Trudinger and Beckner-Onofri's inequalities on the CR sphere, Ann. of Math. 177, 1 (2013), 1-52.

[7] D. CAO, Nontrivial solution of semilinear elliptic equation with critical exponent in $\mathbb{R}^{2}$, Comm. Partial Differential Equations 17, 3 (1992), 407-435.

[8] W. S. Cohn AND G. Lu, Best constants for Moser-Trudinger inequalities on the Heisenberg group, Indiana Univ. Math. J. 50, 4 (2001), 1567-1591.

[9] W. S. CoHN AND G. LU, Sharp constants for Moser-Trudinger inequalities on spheres in complex space $C^{n}$, Comm. Pure Appl. Math. 57, 11 (2004), 1458-1493.

[10] S. Y. A. CHANG AND P. YANG, The inequality of Moser and Trudinger and applications to conformal geometry. Dedicated to the memory of Jürgen K. Moser, Comm. Pure Appl. Math. 56, 8 (2003), 11351150.

[11] J. M. Do Ó, N-Laplacian equations in $\mathbb{R}^{n}$ with critical growth, Abstr. Appl. Anal. 2, 3 (1997), 301315.

[12] M. DE Souza And J. M. Do Ó, On singular Trudinger-Moser type inequalities for unbounded domains and their best exponents, Potential Anal. 38, 4 (2013), 1091-1101. 
[13] M. Dong, N. Lam And G. Lu, Sharp weighted Trudinger-Moser and Caffarelli-Kohn-Nirenberg inequalities and their extremal functions, preprint.

[14] M. Dong ANd G. Lu, Best constants and existence of maximizers for weighted Moser-Trudinger inequalities, Calc. Var. Partial Differential Equations 55, 4 (2016), 55-88.

[15] L. Fontana, Sharp borderline Sobolev inequalities on compact Riemannian manifolds, Comment. Math. Helv. 68, 3 (1993), 415-454.

[16] N. LAM AND G. Lu, Sharp constants and optimizers for a class of the Caffarelli-Kohn-Nirenberg inequalities, arXiv:1510.01224, to appear in Advanced Nonlinear Studies.

[17] N. LAM AND G. Lu, Sharp Moser-Trudinger inequality on the Heisenberg group at the critical case and applications, Adv. Math. 231, 6 (2012), 3259-3287.

[18] N. LAM AND G. LU, A new approach to sharp Moser-Trudinger and Adams type inequalities: a rearrangement-free argument, J. Differential Equations 255, 3 (2013), 298-325.

[19] N. LAM AND G. LU, $N$-Laplacian equations in $R^{N}$ with subcritical and critical growth without the Ambrosetti-Rabinowitz condition, Adv. Nonlinear Stud. 13, 2 (2013), 289-308.

[20] N. LAM, G. LU AND H. TANG, Sharp subcritical Moser-Trudinger inequalities on Heisenberg groups and subelliptic PDEs, Nonlinear Anal. 95, (2014), 77-92.

[21] N. LAM, G. LU AND H. TANG, On nonuniformly subelliptic equations of Q-sub-Laplacian type with critical growth in the Heisenberg group, Adv. Nonlinear Stud. 12, 3 (2012), 659-681.

[22] N. LAM, G. LU AND L. ZHANG, Equivalence of critical and subcritical sharp Trudinger-MoserAdams inequalities, arXiv:1504.04858, to appear in Revista Matematica Iberoamericana.

[23] Y. X. LI, Remarks on the extremal functions for the Moser-Trudinger inequality, Acta Math. Sin. (Engl. Ser.) 22, 2 (2006), 545-550.

[24] Y. X. LI, Moser-Trudinger inequality on compact Riemannian manifolds of dimension two, J. Partial Differential Equations 14, 2 (2001), 163-192.

[25] Y. X. LI AND B. RUF, A sharp Moser-Trudinger type inequality for unbounded domains in $\mathbb{R}^{n}$, Indiana Univ. Math. J. 57, 1 (2008), 451-480.

[26] G. Lu AND H. TANG, Sharp Moser-Trudinger inequalities on hyperbolic spaces with exact growth condition, J. Geom. Anal. 26, 2 (2016), 837-857.

[27] G. LU AND H. TANG, Sharp singular Trudinger-Moser inequalities in Lorentz-Sobolev spaces, Adv. Nonlinear Stud. 16, 3 (2016), 581-601.

[28] G. Lu AND H. TANG, Best constants for Moser-Trudinger inequalities on high dimensional hyperbolic spaces, Adv. Nonlinear Stud. 13, 4 (2013), 1035-1052.

[29] G. LU, H. TANG AND M. ZHU, Best constants for Adams' inequalities with the exact growth condition in $R^{n}$, Adv. Nonlinear Stud. 15, 4 (2015), 763-788.

[30] N. Masmoudi AND F. SANi, Adams' inequality with the exact growth condition in $\mathbb{R}^{4}$, Comm. Pure Appl. Math. 67, 8 (2014), 1307-1335.

[31] N. Masmoudi And F. SAni, Trudinger-Moser inequalities with the exact growth condition in $\mathbb{R}^{n}$ and application, Comm. Partial Differential Equations 40, 8 (2015), 1408-1440.

[32] J. Moser, A sharp form of an inequality by N. Trudinger, Indiana Univ. Math. J. 20, (1970/71), 1077-1092.

[33] S. I. Pohozaev, On the eigenfunctions of the equation $\Delta u+\lambda f(u)=0$, (Russian), Dokl. Akad. Nauk SSSR. 165 (1965), 36-39.

[34] B. RuF, A sharp Moser-Trudinger type inequality for unbounded domains in $\mathbb{R}^{2}$, J. Funct. Anal. 219, 2 (2005), 340-367.

[35] N. S. Trudinger, On imbeddings into Orlicz spaces and some applications, J. Math. Mech. 17 (1967), 473-483.

[36] V. I. Yudovich, Some estimates connected with integral operators and with solutions of elliptic equations, (Russian), Dokl. Akad. Nauk SSSR. 138 (1961), 805-808.

[37] J. ZHU, Improved Moser-Trudinger inequality involving $L^{p}$ norm in n-dimensions, Adv. Nonlinear Stud. 14, 2 (2014), 273-293. 